\title{
Role of Epidemic Model to Control Drinking Problem
}

\author{
Ankit Agrawal ${ }^{1 *}$, Abha Tenguria ${ }^{2}$, Geeta Modi ${ }^{1}$ \\ ${ }^{1}$ Department of Mathematics, Govt. Motilal Vigyan Mahavidyalya, Bhopal (M.P.), India \\ ${ }^{2}$ Department of Mathematics, Govt. Maharani Laxmi Bai Girls (autonomous) P. G. Collage, Bhopal (M.P.), India \\ ${ }^{1}$ Department of Mathematics, Govt. Motilal Vigyan Mahavidyalya, Bhopal (M.P.), India \\ *Corresponding Author: ankit_agrawal458@yahoo.com, Tel.: +91-9926958880
}

Available online at: www.isroset.org

Accepted 17/Aug/2018, Online 30/Aug/2018

\begin{abstract}
In this paper, we have developed a non-linear SHTR mathematical model of alcohol abuse with non-linear incidence rate proposed by Anderson and May [1] which consists of four compartments corresponding to four population classes, namely, non- drinkers $(S)$, heavy drinkers $(H)$, drinkers in treatment $(T)$ and temporarily recovered class $(R)$. The basic properties and sensitivity analysis of the system are discussed. Next, Basic reproduction number $R_{0}$ is calculated. The local and global stability of the drinking-free (problem free) equilibrium $E_{0}$ and the endemic equilibrium $E^{*}$ of the model are discussed. The local asymptotical stability of equilibrium is verified by analyzing the eigenvalues and using the Routh-Hurwitz criterion. Also discuss the global asymptotical stability of the drinking-free equilibrium by using LaSalle's invariance principle and endemic equilibrium by autonomous convergence theorem. The stability analysis of the model shows that the system is locally asymptotically stable at drinking-free equilibrium $E_{0}$ when $R_{0}<1$. When $R_{0}>1$, endemic equilibrium $E^{*}$ exists and the system becomes locally asymptotically stable at $E^{*}$ and $E_{0}$ becomes unstable. Finally, numerical findings by using actual data of my village are illustrated through computer simulations using MATLAB software, which show the reducing the contact rate between the non-drinkers and heavy drinkers, increasing the number of drinkers that go into treatment and awareness combating the drinking epidemic.
\end{abstract}

Keywords - epidemic, treatment, awareness effect, drinking-free and endemic equilibrium, local and global stability.

Mathematics Subject Classification (2010): 92D25, 92D30, 93B35.

\section{INTRODUCTION}

Nowadays alcoholism has become an epidemic disease. Alcoholism is generally used to mean compulsive and uncontrolled consumption of alcoholic beverage which affects their work, health, education and social life. Alcohol abuse and alcoholism are two different forms of problems of drinking. Alcohol abuse is when alcohol drinking leads problems, but not physical addiction. On the other hand alcoholism is when one has signs of physical addiction to alcohol and continues to drink, despite problems with physical health, mental health, family or job responsibilities. In fact alcoholism is a long term effect of alcohol abuse. Alcohol abuse and alcoholism can affect all aspects of our life. Long-term alcohol use can cause serious health complications, damaging nearly every organ and system in the body including our brain.

Alcohol is the world's third leading cause of ill health and premature death, after low birth weight and unsafe sex. World Health Organization (WHO) estimates that alcohol is supposed to cause about 60 types of diseases and injury like 20-30\% of esophageal cancer, liver cancer, cirrhosis of the liver, homicide, epileptic seizures and motor vehicle accidents worldwide. Over-consumption of alcohol is now the third leading cause of death all over the world. Alcohol related problems cost so much that it affects the economic structure of the countries $[3,4]$.

Individuals who wish to overcome an alcohol abuse problem can enter into the treatment programmes. Completely stopping the use of alcohol is the ideal goal of treatment. This is called abstinence. Completely stopping or avoiding alcohol is difficult for many people with alcoholism. Most of them seek outside help from treatment centers and therapy sessions. These 
programmes usually offer counselling and therapy to discuss alcoholism and its effects, mental health support, medical care etc. There are two major forms of intervention policy of alcohol abuse: (i) prevention initiation into alcohol abuse and (ii) rehabilitation of alcohol abusers. Among the many problems confronting these programmes, the most important is the very high rates of relapse after treatment. The National Institute on Alcohol Abuse and Alcoholism estimates that up to $70 \%$ of treated alcohol abusers relapse after treatment which is indeed a big problem. Therefore prevention and control efforts that include treatment and education (Awareness) programmes should be improved so that the rate of relapse from treatment can be reduced.

It is obvious that alcohol abuse and alcoholism not only causes health problems but also has great social and economic impacts on the countries. Therefore, it is very important to understand the dynamics of alcoholism spread among the populations and identify the parameters of greater importance which will help the policy-makers in targeting prevention and treatment resources for maximum effectiveness. Although drinking is a problem of significant public health importance, not much has been analyzed in terms of using mathematical modelling to gain insight into its transmission dynamics at population level. Most of the existing works on alcohol abuse and alcoholism are of clinical aspects.

Mathematical models could mimic the process of drinking and provided useful tools to analyze the spread and control of drinking behaviour. Several different mathematical models for drinking had been formulated and studied [2, 4, 5, 6, 7, 8, 9, 10]. Giuseppe and Brian [4] developed a two-stage (four components) model for youths with serious drinking problems and their treatment. The stability of all the equilibria was analyzed. Mubayi et al. [2] introduced a simple framework where drinking was modeled as a socially contagious process in low and high-risk connected environments. Lee et al. [5] introduced a mathematical model of drinking that incorporated the impact of relapse, and was analyzed primarily under the impact of two time-dependent controls put in place over a infinite time horizon. Xiang et al. [6] presented a quit drinking model taking into account of permanent quit drinker compartment and relapse, and global stability of equilibria was obtained. Huo and Song [7] introduced a more realistic two-stage model for binge drinking problem, where the youths with alcohol problems were divided into those who admitted the problem and those who did not admit. Mathematical analyses established that the global dynamics of the model were determined by the basic reproduction number. Swarnali Sharma [8] and Isaac Kwasi Adu [9] introduce four compartmental models with treatment and relapse. For the other mathematical models for drinking, we referred to [10] and the references therein.

The organization of the paper is as follows. In section-III, 3.1 describe the development of model and give a detail of model assumptions, 3.2 presented basic properties of the system, and 3.3 presented the basic reproduction and give a detail of sensitivity analysis of reproduction in 3.4. The detail of equilibrium points presented in 3.5. The local stability of the system presented in 3.6 and the global stability of the system presented in 3.7. A numerical simulation experiment has been presented in Section-IV. Conclusion and future scope presented in the section-V.

\section{RELATED WORK}

Motivated by the work of Swarnali Sharma [8] and Isaac Kwasi Adu [9], in this paper, an SHTR epidemic model included with the effect of saturated incidence rate $g(S) H=\frac{\beta S H}{1+\alpha S}$ concerned. This rate was proposed by Anderson and May [1]. The purpose of this paper is to show that the effect of treatment and awareness parameter to control drinking problem.

\subsection{The Mathematical model}

\section{Methodology}

\subsubsection{Model description}

Formulate a mathematical model and divide the population into four compartments: non-drinkers $(S)$, heavy drinkers $(H)$, drinkers in treatment $(T)$, and recovered drinkers $(R)$. The interactions between the four drinking states are shown in the schematic diagram in figure 1. 


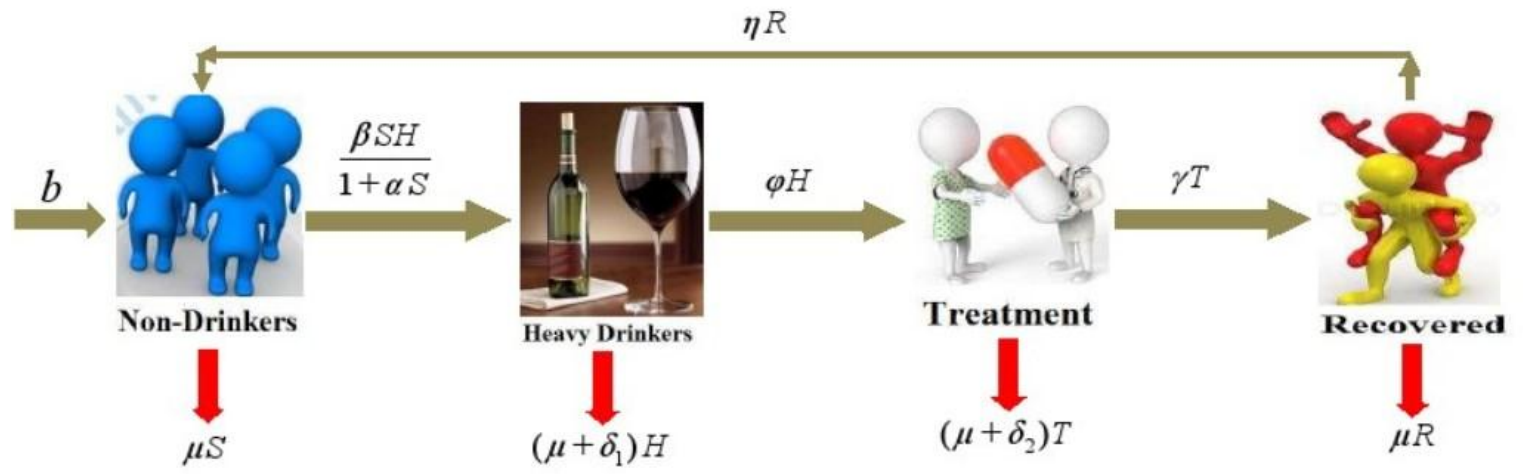

Figure 1. Schematic diagram of the alcohol abuse model

\subsubsection{Model assumptions}

The following assumptions were made in the model:

(i) The drinking epidemic occurs in a closed environment.

(ii) Farmers, labors and sex workers taking wine every evening for body pain relief, mental peace and for sleep.

(iii) Social status and race do not affect the probability of becoming a heavy drinker.

(iv) Heavy drinking is transmitted to non-drinkers when they are in contact with heavy drinkers.

(v) Members mix homogeneously (have the same interaction to the same degree).

(vi) Drinkers in treatment may only become heavy drinkers again after passing through the recovery and susceptible compartments respectively.

(vii) Drinkers who have stopped drinking enter into recovery compartment.

Under the above assumptions the SIR epidemic model takes the following form:

$$
\left.\begin{array}{rl}
\frac{d S}{d t} & =b-\frac{\beta S H}{1+\alpha S}-\mu S+\eta R \\
\frac{d H}{d t} & =\frac{\beta S H}{1+\alpha S}-\left(\mu+\delta_{1}+\phi\right) H \\
\frac{d T}{d t} & =\phi H-\left(\mu+\delta_{2}+\gamma\right) T \\
\frac{d R}{d t} & =\gamma T-(\mu+\eta) R
\end{array}\right\}
$$

with the initial conditions $S(0) \geq 0, H(0) \geq 0, T(0) \geq 0$ and $R(0) \geq 0$.

Here, $b$ is the recruitment rate of $S, \alpha$ is the awareness effect parameter, $\beta$ is transmission rate from $S$ to $H, \eta$ is the transmission rate from $R$ to $H, \mu$ is natural death rate, $\delta_{1}$ is the drinking induced death rate of $H, \delta_{2}$ is the drinking induced death rate of $T, \phi$ is proportion of drinkers entering $T$ compartment and $\gamma$ is the recovered rate of $T$. $b, \alpha, \beta, \eta$, $\mu, \delta_{1}, \delta_{2}, \phi$ and $\gamma$ all are positive.

In the model (1), incidence rate $g(S) H=\frac{\beta S H}{1+\alpha S} \rightarrow \frac{\beta}{\alpha} H$ as $S \rightarrow \infty$ i.e. $g(S)$ tends to a saturation level when $S$ gets large. Parameter $\alpha$ measures the awareness effect of susceptible for epidemic control. 


\subsection{Basic Properties}

\subsubsection{Non-negative of solutions}

Theorem 3.2.1 Every solution of system (1) with initial conditions (2) exists in the interval $[0, \infty)$ and $S(t) \geq 0, H(t) \geq 0$, $T(t) \geq 0$ and $R(t) \geq 0$ for all $\mathrm{t} \geq 0$.

Proof. Since the right hand side of system (1) is completely continuous and locally Lipschitzian on C (space of continuous functions), the solution $(S(t), H(t), T(t), R(t))$ of (1) with initial conditions (2) exists and is unique on [0, $\xi$ ), where $0<\xi \leq+\infty$. From the second equation of (1), we have

$$
H(t)=H(0) \exp \left[\int_{0}^{t}\left\{\frac{\beta S}{1+\alpha S}-\left(\mu+\delta_{1}+\phi\right)\right\} d s\right] \geq 0
$$

From the third equation of (1) have

$$
\begin{aligned}
& \frac{d T}{d t} \geq-\left(\mu+\delta_{2}+\gamma\right) T(t) \quad[\because H(t) \geq 0] \\
& \Rightarrow T(t) \geq T(0) \exp \left[-\left(\mu+\delta_{2}+\gamma\right) t\right] \geq 0
\end{aligned}
$$

Similarly, from the forth equation of (1) have

$$
\begin{aligned}
& \frac{d R}{d t} \geq-(\mu+\eta) R(t) \quad[\because T(t) \geq 0] \\
& \Rightarrow R(t) \geq R(0) \exp [-(\mu+\eta) t] \geq 0 .
\end{aligned}
$$

Finally, it follows from the first equation of the system (1) that,

$$
\frac{d S}{d t} \geq b-\left[\frac{\beta H}{1+\alpha S}+\mu\right] S(t) \quad[\because R(t) \geq 0] .
$$

Thus,

$$
\frac{d}{d t}\left[S(t) \exp \left\{\mu t+\int_{0}^{t} \frac{\beta H(s)}{1+\alpha S} d s\right\}\right] \geq b \exp \left\{\mu t+\int_{0}^{t} \frac{\beta H(s)}{1+\alpha S} d s\right\} .
$$

Hence,

so that

$$
S(t) \exp \left\{\mu t+\int_{0}^{t} \frac{\beta H(s)}{1+\alpha S} d s\right\}-S(0) \geq \int_{0}^{t} b \exp \left\{\mu t+\int_{0}^{t} \frac{\beta H(s)}{1+\alpha S} d s\right\} d t
$$

$$
S(t) \geq S(0) \exp \left[-\left\{\mu t+\int_{0}^{t} \frac{\beta H(s)}{1+\alpha S} d s\right\}\right]+\exp \left\{-\left(\mu t+\int_{0}^{t} \frac{\beta H(s)}{1+\alpha S} d s\right)\right\} \times\left[\int_{0}^{t} b \exp \left\{\mu t+\int_{0}^{t} \frac{\beta H(s)}{1+\alpha S} d s\right\} d t\right]>0
$$

$\therefore S(t) \geq 0, H(t) \geq 0, T(t) \geq 0$ and $R(t) \geq 0 \forall \mathrm{t} \geq 0$.

This completes the proof.

\subsubsection{Invariant region}

Theorem 3.2.2 The feasible region $\Gamma=\left\{(S(t), H(t), T(t), R(t)) \in \square_{+}^{4}: S+H T+R \leq \frac{b}{\mu}\right\}$ with initial conditions $S(t)>0$, $H(t)>0, T(t)>0, R(t)>0$ is positively invariant.

Proof. Adding the equations of the system (1), obtain

$$
\begin{aligned}
\frac{d N}{d t} & =\frac{d S}{d t}+\frac{d H}{d t}+\frac{d T}{d t}+\frac{d R}{d t}=b-\mu N-\left(\delta_{1} H+\delta_{2} T\right) \\
\frac{d N}{d t} \leq b-\mu N & {[\because H(t) \geq 0, T(t) \geq 0] }
\end{aligned}
$$

The solution $N(t)$ of the differential equation (3) has the following property,

$$
0<N(t) \leq N(0) e^{-\mu t}+\frac{b}{\mu}\left(1-e^{-\mu t}\right)
$$


where, $N(0)$ represents the sum of the initial values of the variables.

As $t \rightarrow \infty, 0<N \leq \frac{b}{\mu}$. So, if $N(0) \leq \frac{b}{\mu}$, then $\lim _{t \rightarrow \infty} N(t)=\frac{b}{\mu}$. This means that $\frac{b}{\mu}$ is the upper bound of $N$. On the other hand, if $N(0)>\frac{b}{\mu}$, then $N(t)$ will decrease to $\frac{b}{\mu}$. This means that if $N(0)>\frac{b}{\mu}$, then the solution $(S(t), H(t), T(t), R(t))$ enters $\Gamma$ or approach it asymptotically. Hence it is positively invariant under the flow induced by the system (1). Thus in $\Gamma$, the model (1) is well-posed epidemiologically and mathematically. Hence it is sufficient to study the dynamics of the model in $\Gamma$.

\subsection{The basic reproduction number $\boldsymbol{R}_{0}$}

Basic reproduction number [3] for drinking epidemic model is defined as the number of heavy drinkers produced when a single heavy drinker is introduced into susceptible (non-drinkers) population, i.e.,

$R_{0}=$ (effective contact rate) $\times$ (duration of heavy drinkers spends in the drinking class).

In the present model (1), $\frac{b \beta}{(\mu+b \alpha)}$ is the effective contact rate and $\left(\mu+\delta_{1}+\gamma\right)$ is the removal rate of the heavy drinkers from drinking class. By assumption all rates are constant. This means that the expected duration of heavy drinkers spend in the drinking class is simply the inverse of the removal rate, i.e. $\frac{1}{\left(\mu+\delta_{1}+\gamma\right)}$. Therefore, the basic reproduction number of system (1) is given by $R_{0}=\frac{b \beta}{(\mu+b \alpha)\left(\mu+\delta_{1}+\gamma\right)}$.

\subsection{Sensitivity analysis of $\boldsymbol{R}_{0}$}

The basic reproduction number $R_{0}$ of system (1) depends on five parameters, namely, the transmission coefficient from susceptible to heavy drinkers $\beta$, drinking related death rate of heavy drinkers $\delta_{1}$, the pro- portion of drinkers who enter into treatment $\gamma, \alpha$ awareness effect parameter and the natural death rate of population $\mu$. Among those parameters, we cannot control the natural death rate of population $\mu$. Therefore, to examine the sensitivity of $R_{0}$ to the parameters $\beta, \delta_{1}, \gamma$ and $\alpha$, normalized forward sensitivity index with respect to each of those parameters are computed as follows:

$$
\begin{aligned}
& A_{\beta}=\frac{\frac{\partial R_{0}}{R_{0}}}{\frac{\partial \beta}{\beta}}=\frac{\beta}{R_{0}} \frac{\partial R_{0}}{\partial \beta}=\left\{\frac{\beta(\mu+b \alpha)\left(\mu+\delta_{1}+\gamma\right)}{b \beta}\right\}\left\{\frac{b}{(\mu+b \alpha)\left(\mu+\delta_{1}+\gamma\right)}\right\}=1 \\
& A_{\alpha}=\frac{\frac{\partial R_{0}}{\frac{R_{0}}{\alpha}}}{\frac{\partial}{\alpha}}=\frac{\alpha}{R_{0}} \frac{\partial R_{0}}{\partial \alpha}=\frac{-b \alpha}{\left(\mu+\delta_{1}+\gamma\right)} \Rightarrow\left|A_{\alpha}\right|<1, A_{\delta_{1}}=\frac{\frac{\partial R_{0}}{\frac{R_{0}}{\partial \delta_{1}}}}{\delta_{1}} \frac{\delta_{1}}{R_{0}} \frac{\partial R_{0}}{\partial \delta_{1}}=\frac{-\delta_{1}}{\left(\mu+\delta_{1}+\gamma\right)} \Rightarrow\left|A_{\delta_{1}}\right|<1, \\
& A_{\gamma}=\frac{\frac{\partial R_{0}}{\frac{R_{0}}{\gamma}}}{\frac{\partial \gamma}{\gamma}}=\frac{\gamma}{R_{0}} \frac{\partial R_{0}}{\partial \gamma}=\frac{-\gamma}{\left(\mu+\delta_{1}+\gamma\right)} \Rightarrow\left|A_{\gamma}\right|<1 .
\end{aligned}
$$

From the above discussion it is clear that the basic reproduction number $R_{0}$ is most sensitive to changes in $\beta$, the transmission coefficient from susceptible population to heavy drinkers. If $\beta$ will increase $R_{0}$ will increase in same proportion and if $\beta$ will decrease $R_{0}$ will also decrease in same proportion. On the other hand $\delta_{1}, \alpha$ and $\gamma$ have an inversely proportional relationship with $R_{0}$, i.e., an increase in any of them will cause a decrease in $R_{0}$ and a decrease in any of them will cause an increase in $R_{0}$. But the increase in $\delta_{1}$, the drinking related death rate of the heavy drinkers not in treatment, is neither ethical nor practical. So, it is better to focus either on $\beta$, the transmission rate from susceptible population to heavy drinker, $\alpha$ awareness parameter or $\gamma$, the proportion of drinkers who enter into treatment. As $R_{0}$ is more sensitive to changes in $\beta$ than 
$\alpha$ and $\gamma$, it seems sensible to focus on the reduction of $\beta$ and $\alpha$ is increase to control the alcohol abuse. This sensitivity analysis tells us that efforts to increase prevention are more effective in controlling the spread of alcohol abuse in population than efforts to increase the numbers of heavy drinkers accessing treatment.

\subsection{Equilibrium points and existence}

In this section, the drinking-free (problem free) and the endemic equilibrium points of system (1) find and analyze their existence.

The equilibrium points of the model system (1) are:

1. Drinking-free (problem free) equilibrium: $E_{0}\left(\frac{b}{\mu}, 0,0,0\right)$,

2. Endemic equilibrium: $E^{*}\left(S^{*}, H^{*}, T^{*}, R^{*}\right)$,

Here use the term "drinking-free equilibrium" to describe the state where a drinking culture does not exist, i.e. the equilibrium points of system $(1)$ at the origin $(0,0)$. On the other hand, "endemic equilibrium" stands for the state where a drinking culture exists, i.e. the non- trivial positive solution of system (1).

\subsubsection{Existence of epidemic equilibrium $E^{*}\left(S^{*}, H^{*}, T^{*}, R^{*}\right)$}

At an endemic equilibrium, drinkers are present and the following conditions hold:

$S>0, H>0, T>0$ and $R>0$.

$$
b-\frac{\beta S H}{1+\alpha S}-\mu S+\eta R=0, \frac{\beta S H}{1+\alpha S}-\left(\mu+\delta_{1}+\phi\right) H=0, \phi H-\left(\mu+\delta_{2}+\gamma\right) T=0, \gamma T-(\mu+\eta) R=0 .
$$

By some simple calculation, get

$$
S^{*}=\frac{\mu+\delta_{1}+\phi}{\beta-\alpha\left(\mu+\delta_{1}+\phi\right)}, T^{*}=\frac{\phi H}{\left(\mu+\delta_{2}+\gamma\right)}, \mathrm{R}^{*}=\frac{\phi \gamma H}{(\mu+\eta)\left(\mu+\delta_{2}+\gamma\right)}
$$

and $H^{*}=\frac{(b \alpha+\mu)\left(\mu+\delta_{1}+\phi\right)\left[R_{0}-1\right]}{[\beta-1]\left[\beta-\alpha\left(\mu+\delta_{1}+\phi\right)\right]\left[(\mu+\eta)\left(\mu+\delta_{2}+\gamma\right)\right]-\eta \phi \gamma\left[\beta-\alpha\left(\mu+\delta_{1}+\phi\right)\right]}$.

By applying the Descarte's rule of signs if $R_{0}>1$, one positive equilibria exists and if $R_{0}<1$, system has no positive equilibrium.

Summarizing the previous discussions come to the following result:

Theorem 3.5.1 The system (1) has a drinking- free equilibrium $E_{0}\left(\frac{b}{\mu}, 0,0,0\right)$, which exists for all parameter values. If $R_{0}>1$, the system (1) also admits a unique endemic equilibrium $E^{*}\left(S^{*}, H^{*}, T^{*}, R^{*}\right)$. If $R_{0}<1$, then the system has no endemic equilibrium and if $R_{0}>1$, system has a positive equilibrium.

\subsection{Local stability analysis}

\subsubsection{Local stability of drinking-free (problem free) equilibrium}

In this section find the local stability of the system (1) at drinking-free equilibrium $E_{0}$.

Let system (1) as:

$$
\begin{aligned}
& \frac{d S}{d t}=b-\frac{\beta S H}{1+\alpha S}-\mu S+\eta R \equiv f_{1}, \frac{d H}{d t}=\frac{\beta S H}{1+\alpha S}-\left(\mu+\delta_{1}+\phi\right) H \equiv f_{2} \\
& \frac{d T}{d t}=\phi H-\left(\mu+\delta_{2}+\gamma\right) T \equiv f_{3}, \frac{d R}{d t}=\gamma T-(\mu+\eta) R \equiv f_{4}
\end{aligned}
$$

Then the Jacobian matrix is 


$$
J(S, H, T, R)=\left[\begin{array}{cccc}
-\frac{\beta H}{(1+\alpha S)^{2}}-\mu & -\frac{\beta S}{1+\alpha S} & 0 & \eta \\
\frac{\beta H}{(1+\alpha S)^{2}} & \frac{\beta S}{1+\alpha S}-\left(\mu+\delta_{1}+\phi\right) & 0 & 0 \\
0 & \phi & -\left(\mu+\delta_{2}+\gamma\right) & 0 \\
0 & 0 & \gamma & -(\gamma+\eta)
\end{array}\right]
$$

The Jacobian matrix of system (1) at $E_{0}\left(\frac{b}{\mu}, 0,0,0\right)$ is

$$
J\left(E_{0}\right)=\left[\begin{array}{cccc}
-\mu & -\frac{\beta b}{\alpha+\alpha b} & 0 & \eta \\
0 & \frac{\beta b}{\alpha+\alpha b}-\left(\mu+\delta_{1}+\phi\right) & 0 & 0 \\
0 & \phi & -\left(\mu+\delta_{2}+\gamma\right) & 0 \\
0 & 0 & \gamma & -(\gamma+\eta)
\end{array}\right]
$$

Then the characteristic equation of the Jacobian matrix $J\left(E_{0}\right)$ is

$$
(\lambda+\mu)\left(\frac{\beta b}{\alpha+\alpha b}-\left(\mu+\delta_{1}+\phi\right)-\lambda\right)\left(\mu+\delta_{2}+\gamma+\lambda\right)(\gamma+\eta+\lambda)=0 .
$$

Therefore, eigenvalues of the characteristic equation $J\left(E_{0}\right)$ are

$$
\lambda_{1}=-\mu, \lambda_{2}=\frac{\beta b}{\alpha+\alpha b}-\left(\mu+\delta_{1}+\phi\right), \lambda_{3}=-\left(\mu+\delta_{2}+\gamma\right) \text { and } \lambda_{4}=-(\gamma+\eta) .
$$

Here, $\lambda_{1}, \lambda_{3}$ and $\lambda_{4}$ are clearly real and negative. Now, $E_{0}$ is stable if $\lambda_{2}$ is negative if $\frac{\beta b}{\alpha+\alpha b}-\left(\mu+\delta_{1}+\phi\right)<0$, i.e., $R_{0}<1$. So all eigenvalues are negative if $R_{0}<1$, and hence $E_{0}$ is local asymptotically stable. If $R_{0}=1$, then $\lambda_{2}=0$ and $E_{0}$ is locally stable. If $R_{0}>1$, then $\lambda_{2}>0$ which means that there exist a positive eigenvalue. So, $E_{0}$ is unstable. So, the following result obtained:

Theorem 3.6.1 The drinking-free equilibrium $E_{0}$ of the model system (1) is locally asymptotically stable if $R_{0}<1$. If $R_{0}=1$, $E_{0}$ is locally stable. If $R_{0}>1, E_{0}$ is unstable.

\subsubsection{Local stability of endemic equilibrium}

In this section find the local stability of the system (1) at endemic equilibrium $E^{*}\left(S^{*}, H^{*}, T^{*}, R^{*}\right)$.

The Jacobian matrix of system (1) at $E^{*}\left(S^{*}, H^{*}, T^{*}, R^{*}\right)$ is

$$
J\left(E^{*}\right)=\left[\begin{array}{cccc}
-\frac{\beta H^{*}}{\left(1+\alpha S^{*}\right)^{2}}-\mu & -\frac{\beta S^{*}}{1+\alpha S^{*}} & 0 & \eta \\
\frac{\beta H^{*}}{\left(1+\alpha S^{*}\right)^{2}} & \frac{\beta S^{*}}{1+\alpha S^{*}}-\left(\mu+\delta_{1}+\phi\right) & 0 & 0 \\
0 & \phi & -\left(\mu+\delta_{2}+\gamma\right) & 0 \\
0 & 0 & \gamma & -(\gamma+\eta)
\end{array}\right]
$$

Let $A_{11}=-\frac{\beta H^{*}}{\left(1+\alpha S^{*}\right)^{2}}-\mu, A_{12}=-\frac{\beta S^{*}}{1+\alpha S^{*}}, A_{13}=0, A_{14}=\eta, A_{21}=\frac{\beta H^{*}}{\left(1+\alpha S^{*}\right)^{2}}, A_{22}=\frac{\beta S^{*}}{1+\alpha S^{*}}-\left(\mu+\delta_{1}+\phi\right), A_{23}=0$, $A_{24}=\eta, A_{31}=0, A_{32}=\phi, A_{33}=-\left(\mu+\delta_{2}+\gamma\right), A_{34}=0, A_{41}=0, A_{42}=0, A_{43}=\gamma, A_{44}=-(\gamma+\eta)$.

Substituting $A_{i j}$ into $J\left(E^{*}\right)$, obtain 


$$
J\left(E^{*}\right)=\left[\begin{array}{llll}
A_{11} & A_{12} & A_{13} & A_{14} \\
A_{21} & A_{22} & A_{23} & A_{24} \\
A_{31} & A_{32} & A_{33} & A_{34} \\
A_{41} & A_{42} & A_{43} & A_{44}
\end{array}\right]
$$

Then the characteristic equation of the Jacobian matrix $J\left(E^{*}\right)$ is

$$
\begin{aligned}
& \lambda^{4}+\left(\text { trace of } J\left(E^{*}\right)\right) \lambda^{3}+\left(A_{12} A_{21}-A_{33} A_{44}-A_{11} A_{44}-A_{22} A_{44}-A_{11} A_{33}-A_{22} A_{33}-A_{11} A_{22}\right) \lambda^{2}+\left(A_{11} A_{33} A_{44}+A_{22} A_{33} A_{44}+\right. \\
& \left.A_{11} A_{22} A_{44}+A_{11} A_{22} A_{33}-A_{12} A_{21} A_{33}-A_{12} A_{21} A_{44}\right) \lambda+\left(A_{12} A_{21} A_{33} A_{44}+A_{14} A_{21} A_{32} A_{43}-A_{11} A_{22} A_{33} A_{44}\right)=0
\end{aligned}
$$

Written as the above equation:

where,

$$
\lambda^{4}+C_{1} \lambda^{3}+C_{2} \lambda^{2}+C_{3} \lambda+C_{4}=0
$$

$$
\begin{aligned}
& C_{1}=A_{11}+A_{22}+A_{33}+A_{44}, C_{2}=A_{12} A_{21}-A_{33} A_{44}-A_{11} A_{44}-A_{22} A_{44}-A_{11} A_{33}-A_{22} A_{33}-A_{11} A_{22}, \\
& C_{3}=A_{11} A_{33} A_{44}+A_{22} A_{33} A_{44}+A_{11} A_{22} A_{44}+A_{11} A_{22} A_{33}-A_{12} A_{21} A_{33}-A_{12} A_{21} A_{44} \text { and } \\
& C_{4}=A_{12} A_{21} A_{33} A_{44}+A_{14} A_{21} A_{32} A_{43}-A_{11} A_{22} A_{33} A_{44} .
\end{aligned}
$$

Using the Routh-Hurwitz criterion [6]. It can be seen that all eigen values of the characteristic equation has negative real part if and only if

$$
C_{1}>0, C_{4}>0, C_{1} C_{2}-C_{3}>0 \text { and }\left(C_{1} C_{2}-C_{3}\right) C_{3}-C_{1}^{2} C_{4}>0 .
$$

Theorem 3.6.2 The endemic equilibrium $E^{*}$ of the model system (1) is locally asymptotically stable if and only if inequalities (5) are satisfied.

\subsection{Global stability analysis}

\subsubsection{Global stability of drinking-free equilibrium}

In this section discuss about the global stability of the drinking free equilibrium $E_{0}$ when $R_{0}^{*}=\frac{\beta b}{(\mu+\alpha b)\left(\mu+\delta_{1}\right)}<1$.

Consider the Lyapunov function as follows:

$$
L=\gamma H+\gamma T+\left(\mu+\delta_{2}+\gamma\right) R
$$

The derivative of $L=(H, T, R)$ with respect to $t$ gives

$$
\begin{aligned}
\frac{d L}{d t} & =\gamma \frac{d H}{d t}+\gamma \frac{d T}{d t}+\left(\mu+\delta_{2}+\gamma\right) \frac{d R}{d t} \\
\frac{d L}{d t} & =\gamma\left(\frac{\beta S H}{1+\alpha S}-\left(\mu+\delta_{1}+\phi\right) H\right)+\gamma\left(\phi H-\left(\mu+\delta_{2}+\gamma\right) T\right)+\left(\mu+\delta_{2}+\gamma\right) \\
& \times(\gamma T-(\mu+\eta) R) \\
& \leq \gamma\left[\frac{\beta S}{1+\alpha S}-\left(\mu+\delta_{1}\right)\right] H \\
& \leq \gamma\left[\frac{\beta b}{\mu+\alpha b}-\left(\mu+\delta_{1}\right)\right] H
\end{aligned}
$$

Now, $\frac{d L}{d t}<0$ if $\frac{\beta b}{\mu+\alpha b}<\left(\mu+\delta_{1}\right)$.

Furthermore, $\frac{d L}{d t}=0$ if and only if $H=0$. Therefore, the largest compact invariant set in $\left\{(S(t), H(t), T(t), R(t)) \in \Gamma: \frac{d l}{d t}=0\right\}$ when $\frac{\beta b}{\mu+\alpha b}<\left(\mu+\delta_{1}\right)$, is the singleton $\left\{E_{0}\right\}$. LaSalle's invariance principle [6] implies that $E_{0}$ is globally asymptotically stable in $\Gamma$ when $\frac{\beta b}{\mu+\alpha b}<\left(\mu+\delta_{1}\right)$ (which implies $\left.R_{0}^{*}<1\right)$. So, arrive to the following result: 
Theorem 3.7.1 If $\frac{\beta b}{\mu+\alpha b}<\left(\mu+\delta_{1}\right)$ then the drinking free equilibrium (DFE) $E_{0}$ of model system (1) is globally asymptotically stable.

\subsubsection{Global stability of endemic equilibrium}

Determine the global stability of the endemic equilibrium in this section.

Reduce the system (1), by using $R(t)=\frac{b}{\mu}-S(t)-H(t)-T(t)$ to eliminate $R(t)$ from the first equation of system (1), which leads to the following three dimensional model:

$$
\begin{aligned}
\frac{d S}{d t} & =b\left(1+\frac{\eta}{\mu}\right)-\frac{\beta S H}{1+\alpha S}-(\mu+\eta) S-\eta H-\eta T \\
\frac{d H}{d t} & =\frac{\beta S H}{1+\alpha S}-\left(\mu+\delta_{1}+\phi\right) H \\
\frac{d T}{d t} & =\phi H-\left(\mu+\delta_{2}+\gamma\right) T
\end{aligned}
$$

in the region $\Gamma=\left\{(S(t), H(t), T(t)) \in \square_{+}^{3}: S+H+T \leq 1\right\}$ with initial conditions $S(t)>0, H(t)>0, T(t)>0$.

Now, use the method of Li and Muldowney [7], the geometric approach method, for the global stability of an endemic equilibrium. We find the sufficient conditions for which the endemic equilibrium is globally asymptotically stable. We first briefly explain the geometric approach method. Consider

$$
x^{\prime}=f(x)
$$

where $f: D \rightarrow R^{n}, D \subset R^{n}$ is an open set and is simply connected and $f \in C^{1}(D)$.

Let $x^{*}$ be the solution of (6) i.e. $f\left(x^{*}\right)=0$. Assume that the following hypotheses hold.

(H1) There exists a compact absorbing set $K \subset D$.

(H2) Equation (7) has a unique equilibrium $x^{*}$ in $D$.

The basic idea of this method is that if the equilibrium $x^{*}$ is locally stable, then the stability is assured provided that $(H 1)$ and (H2) hold and no non constant periodic solution of (7) exists. Therefore, sufficient conditions on $f$ capable of precluding the existence of such solutions have to be detected.

Suppose that assumptions $(H 1)$ and $(H 2)$ hold. Assume that (7) satisfies a Bendixson criterion that is robust under $C^{1}$ local perturbations of $f$ at all non-equilibrium non-wandering points for (7). The $x^{*}$ is globally stable in $D$ provided it is stable.

Let $P(x)$ be a $\left(\begin{array}{l}n \\ 2\end{array}\right) \times\left(\begin{array}{l}n \\ 2\end{array}\right)$ matrix valued function that is $C^{1}$, on $D$ and consider

$$
B=P_{f} P^{-1}+P J^{[2]} P^{-1}
$$

where the matrix $P_{f}$ is

$$
\frac{\partial P_{i j}^{*}}{\partial x} f=\left.\frac{d P_{i j}}{d t}\right|_{(7)}
$$

and the matrix $J^{[2]}$ is the second additive compound matrix of the Jacobian matrix $J$, that is, $J(x)=D f(x)$. Generally speaking for an $n \times n$ matrix $J=\left(J_{i j}\right), J^{[2]}$ is a $\left(\begin{array}{l}n \\ 2\end{array}\right) \times\left(\begin{array}{l}n \\ 2\end{array}\right)$ matrix and in the special case $n=3$ one has

$$
J^{[2]}=\left[\begin{array}{ccc}
J_{11}+J_{22} & J_{23} & -J_{13} \\
J_{32} & J_{11}+J_{33} & J_{12} \\
-J_{31} & J_{21} & J_{22}+J_{33}
\end{array}\right]
$$


Consider the Lozinski $\breve{i}$ measure $\mu$ of $B$ with respect to a vector norm $\|\cdot\|$ in $R^{N}, N=\left(\begin{array}{l}n \\ 2\end{array}\right)$, defined by $\mu(B)=\lim _{h \rightarrow 0^{+}} \frac{\|I+h B\|-1}{h}$

It is proved in [7] that if $(H I)$ and $(H 2)$ hold, condition

$$
q=\lim _{t \rightarrow \infty} \sup _{x_{0} \in K} \frac{1}{t} \int_{0}^{t} \mu\left(B\left(x\left(s, x_{0}\right)\right)\right) d s<0
$$

It is shown in [7] that, if $D$ is simply connected, the condition $q<0$ rules out the presence of any orbit that gives rise to a simple closed rectifiable curve that is invariant for (7), such as periodic orbits, homoclinic orbits, and heteroclinic cycles. Moreover, it is robust under $C^{1}$ local perturbations of $f$ near any non equilibrium point that is non-wandering. In particular, the following global-stability result is proved in Li and Muldowney [7].

Lemma 3.7.2 Assume that $D$ is simply connected and that the assumptions $(H 1)$ and $(H 2)$ hold. Then the unique equilibrium $x^{*}$ of (7) is globally stable in $D$ if $q<0$.

Now, study the global stability of the endemic equilibrium $E^{*}$ and obtain.

Theorem 3.7.2 If $R_{0}>1$ then the endemic equilibrium $E^{*}$ of the system (6) is globally stable.

$$
\begin{gathered}
J(S, H, T)=\left[\begin{array}{ccc}
-\frac{\beta H}{(1+\alpha S)^{2}}-\mu-\eta & -\eta-\frac{\beta S}{1+\alpha S} & \eta \\
\frac{\beta H}{(1+\alpha S)^{2}} & \frac{\beta S}{1+\alpha S}-\left(\mu+\delta_{1}+\phi\right) & 0 \\
0 & \phi & -\left(\mu+\delta_{2}+\gamma\right)
\end{array}\right] \\
J^{[2]}=\left[\begin{array}{ccc}
\left(-\frac{\beta H}{(1+\alpha S)^{2}}+\frac{\beta S}{1+\alpha S}-\right. & 0 & \eta \\
\left.2 \mu-\eta-\delta_{1}-\phi\right) & \frac{\beta H}{(1+\alpha S)^{2}}-\left(2 \mu+\delta_{2}\right. & -\eta-\frac{\beta S}{1+\alpha S} \\
\phi & +\eta+\gamma) & \frac{\beta S}{1+\alpha S}-\left(2 \mu+\delta_{1}\right. \\
0 & \frac{\beta H}{(1+\alpha S)^{2}} & +\gamma+\phi)
\end{array}\right]
\end{gathered}
$$

Choose the function

$$
\begin{aligned}
& P=P(S, H, T)=\operatorname{diag}\left(1, \frac{T}{H}, \frac{T}{H}\right) ; \text { then } \\
& P^{-1}=\operatorname{diag}\left(1, \frac{H}{T}, \frac{H}{T}\right)
\end{aligned}
$$

and

$$
P_{f}=\operatorname{diag}\left(0, \frac{H^{\prime} T-T^{\prime} H}{T^{2}}, \frac{H^{\prime} T-T^{\prime} H}{T^{2}}\right) .
$$

Also we have

$$
P_{f} P^{-1}=\operatorname{diag}\left(0, \frac{H^{\prime}}{H}-\frac{T^{\prime}}{T}, \frac{H^{\prime}}{H}-\frac{T^{\prime}}{T}\right)
$$




$$
P J^{[2]} P^{-1}=\left[\begin{array}{ccc}
\left(-\frac{\beta H}{(1+\alpha S)^{2}}+\frac{\beta S}{1+\alpha S}-\right. & \frac{\eta T}{H} \\
\left.2 \mu-\eta-\delta_{1}-\phi\right) & 0 & \\
\frac{\phi H}{T} & -\frac{\beta H}{(1+\alpha S)^{2}}-\left(2 \mu+\delta_{2}\right. & -\eta-\frac{\beta S}{1+\alpha S} \\
0 & \frac{\beta H}{(1+\alpha S)^{2}} & \frac{\beta S}{1+\alpha S}-\left(2 \mu+\delta_{1}\right. \\
& & +\gamma+\phi)
\end{array}\right]
$$

The matrix $B=P_{f} P^{-1}+P J^{[2]} P^{-1}$ can be written in matrix form

$$
B=\left[\begin{array}{ll}
B_{11} & B_{12} \\
B_{21} & B_{22}
\end{array}\right]
$$

where,

$$
\begin{aligned}
& B_{11}=-\frac{\beta H}{(1+\alpha S)^{2}}+\frac{\beta S}{1+\alpha S}-2 \mu-\eta-\delta_{1}-\phi \quad, \quad B_{12}=\left(0, \frac{\eta T}{H}\right) \quad, \quad \text { and } \\
& B_{22}=\left(\begin{array}{cc}
-\frac{\beta H}{(1+\alpha S)^{2}}-\left(2 \mu+\delta_{2}+\eta+\gamma\right) & -\eta-\frac{\beta S}{1+\alpha S} \\
+\frac{H^{\prime}}{H}-\frac{T^{\prime}}{T} & \frac{\beta S}{1+\alpha S}-\left(2 \mu+\delta_{1}+\gamma+\phi\right) \\
\frac{\beta H}{(1+\alpha S)^{2}} & +\frac{H^{\prime}}{H}-\frac{T^{\prime}}{T}
\end{array}\right)
\end{aligned}
$$

Let $(u, v, w)$ be a vector in $R^{3}$; its norm $\|$.$\| is defined as \|(u, v, w)\|=\max \{|u|,|v|+|w|\}$ Let $\mu(B)$ be the Lozinskii measure with respect to this norm. We choose $\mu(B) \leq \sup \left\{g_{1}, g_{2}\right\}$

where $g_{1}=\mu_{1}\left(B_{11}\right)+\left|B_{12}\right|, g_{2}=\mu_{1}\left(B_{22}\right)+\left|B_{21}\right|,\left|B_{12}\right|,\left|B_{21}\right|$ are matrix norm with respect to $l_{1}$ vector norm and $\mu_{1}$ denotes the Lozinski $\breve{i}$ measure with respect to $l_{1}$ vector norm, then

$$
\begin{aligned}
& \mu_{1}\left(B_{11}\right)=-\frac{\beta H}{(1+\alpha S)^{2}}+\frac{\beta S}{1+\alpha S}-2 \mu-\eta-\delta_{1}-\phi,\left|B_{12}\right|=\max \left(0, \frac{\eta T}{H}\right)=\frac{\eta T}{H},\left|B_{21}\right|=\frac{\phi H}{T} . \\
& \mu_{1}\left(B_{22}\right)=\max \left\{-\left(2 \mu+\delta_{2}+\eta+\gamma\right)+\frac{H^{\prime}}{H}-\frac{T^{\prime}}{T},-\left(2 \mu+\delta_{1}+\gamma+\phi+\eta\right)+\frac{H^{\prime}}{H}-\frac{T^{\prime}}{T}\right\}=-\left(2 \mu+\delta_{2}+\gamma+\eta\right)+\frac{H^{\prime}}{H}-\frac{T^{\prime}}{T} .
\end{aligned}
$$

Therefore,

$$
\left.\begin{array}{l}
g_{1}=\mu_{1}\left(B_{11}\right)+\left|B_{12}\right|=-\frac{\beta H}{(1+\alpha S)^{2}}+\frac{\beta S}{1+\alpha S}-2 \mu-\eta-\delta_{1}-\phi+\frac{\eta T}{H} \\
g_{2}=\mu_{1}\left(B_{22}\right)+\left|B_{21}\right|=-\left(2 \mu+\delta_{2}+\gamma+\eta\right)+\frac{H^{\prime}}{H}-\frac{T^{\prime}}{T}+\frac{\phi T}{H}
\end{array}\right\}
$$

From (1),

$$
\frac{H^{\prime}}{H}=\frac{\beta S}{1+\alpha S}-\left(\mu+\delta_{1}+\phi\right) \quad \text { and } \quad \frac{T^{\prime}}{T}=\frac{\phi H}{T}-\left(\mu+\delta_{2}+\gamma\right)
$$

Then

$$
\begin{aligned}
& g_{1}=\frac{\beta S}{1+\alpha S}-\left(\mu+\delta_{1}+\phi\right)-(\mu+\eta)-\frac{\beta H}{(1+\alpha S)^{2}}+\frac{\eta T}{H} \quad \leq \frac{\beta S}{1+\alpha S}-\left(\mu+\delta_{1}+\phi\right)-(\mu+\eta)=\frac{H^{\prime}}{H}-(\mu+\eta) \\
& g_{2}=-\left(2 \mu+\delta_{2}+\gamma+\eta\right)+\frac{\beta S}{1+\alpha S}-\frac{\phi H}{T}+\frac{\phi T}{H}=\frac{\beta S}{1+\alpha S}-\left(\mu+\delta_{1}+\phi\right)-(\mu+\eta)=\frac{H^{\prime}}{H}-(\mu+\eta)
\end{aligned}
$$


Furthermore,

$$
\begin{aligned}
\mu(B) & \leq \sup \left\{g_{1}, g_{2}\right\} \\
& \leq\left\{\frac{H^{\prime}}{H}-(\mu+\eta), \frac{H^{\prime}}{H}-(\mu+\eta)\right\} \\
& \leq \frac{H^{\prime}}{H}-(\mu+\eta)
\end{aligned}
$$

By integrating both sides at the same time, obtain

$$
\begin{aligned}
& \frac{1}{t} \int_{0}^{t} \mu(B) \mathrm{ds} \leq-\frac{1}{t} \operatorname{In} \frac{H(t)}{H(0)}-(\mu+\eta) \\
& q=\operatorname{limsupsup}_{t \rightarrow \infty}-\int_{0}^{t} \mu(B) \mathrm{ds}<-(\mu+\eta)<0 .
\end{aligned}
$$

The proof is complete by Lemma 3.7.2.

\section{RESUlTS AND DiSCUSSION}

With the help of MatLab, some numerical results of system (1) are provided to substantiate the analytic results obtained in this section. We choose a set of values of parameters:

Case I. When $b=0.4, \beta=0.7, \mu=0.25, \eta=0.1, \alpha=0.9, \delta_{1}=0.35, \delta_{2}=0.3, \phi=0.7, \gamma=0.09$. It is easy to check that the basic reproduction number $R_{0}=0.665241149<1$ component $S(t)$ approaches to its steady state value while $H(t), T(t)$ and $R(t)$ approach to zero as $t \rightarrow \infty$. This implies that only non-drinkers population is present and the heavy drinkers, drinkers in treatment and recovered drinkers populations reduce to zero $(H=0, T=0, R=0)$. This means that the model at $R_{0}<1$. This also indicates that the drinking free equilibrium $E_{0}$ is asymptotically stable (Figure. 2).

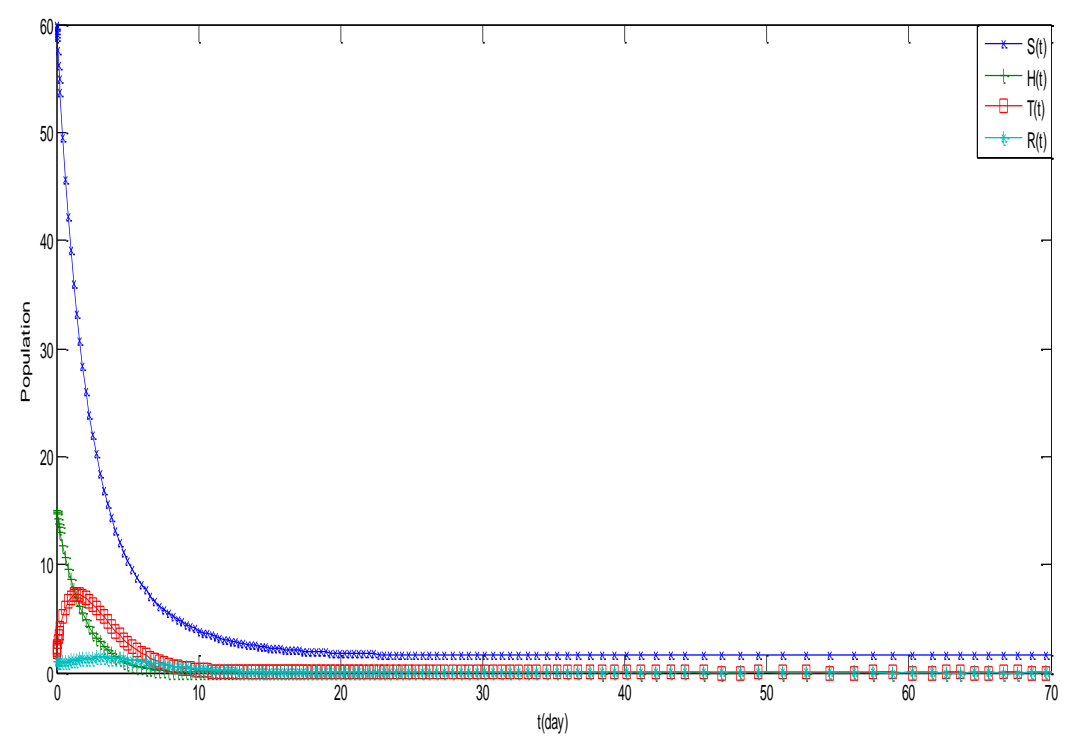

Figure 2. Only non-drinkers exist.

Case II. When $b=0.4, \beta=0.9, \mu=0.25, \eta=0.1, \alpha=0.2, \delta_{1}=0.35, \delta_{2}=0.3, \phi=0.7, \gamma=0.09$ then the basic reproduction number $R_{0}=1.581027668>1$. This shows the non-drinkers $S(t)$, heavy drinkers $H(t)$, drinkers in treatment $T(t)$ and recovered drinkers $R(t)$ coexist in the population. This indicates the existence of drinking problem in the population. People with drinking problem will continue to transform more non-drinkers into heavy drinkers and the drinking -free equilibrium becomes unstable at $R_{0}>1$ (Figure 3). 


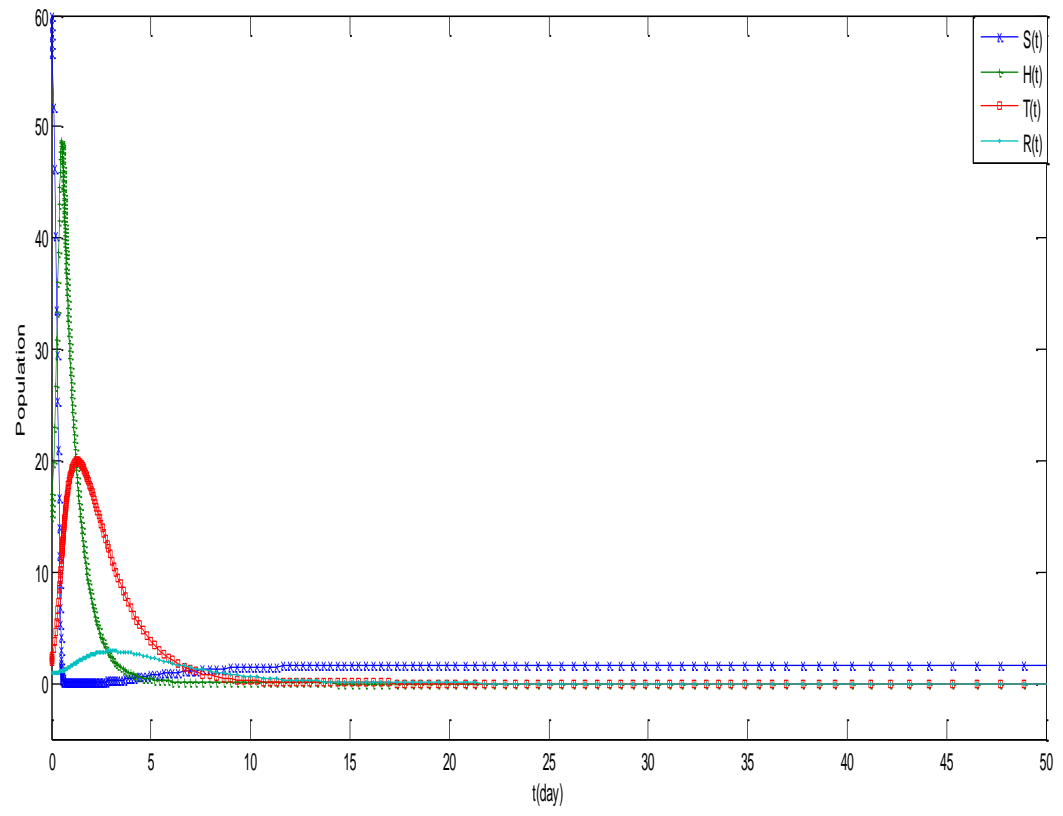

Figure 3. Drinking problem exists.

Case III. When awareness parameter $\alpha$ is increase drinking problem reach under control with respect to time (Figure. 4)

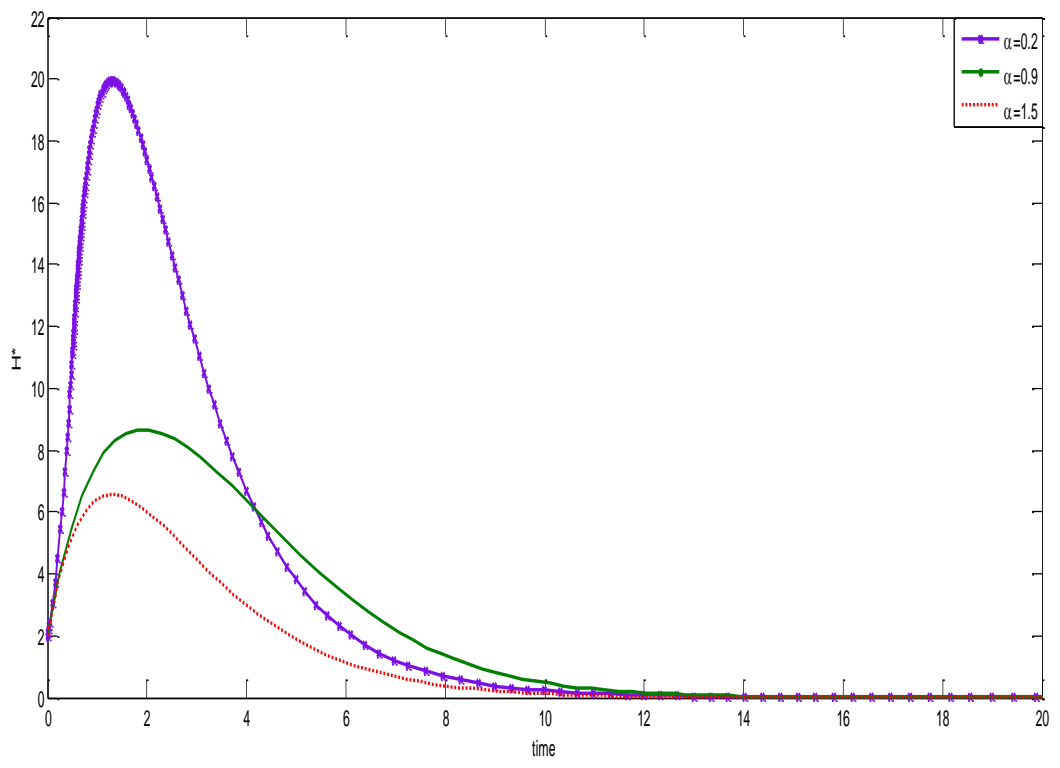

Figure 4. Effect of awareness parameter $\alpha$.

\section{ConCLUSION and Future Scope}

In this chapter, the model shows that, drinking epidemic cannot only be controlled by reducing the contact rate between the nondrinkers and heavy drinkers but also increasing the number of drinkers that go into treatment and aware (educating) drinkers to refrain from drinking can be useful in combating the epidemic. 


\section{REFERENCES}

[1] Adu, K. I. et. al.: "Mathematical Model of Drinking Epidemic", British Journal of Mathematics \& Computer Science 22 (5), pp. 1-10, 2017.

[2] Anderson, R. M. and May, R. M.: "Regulation and Stability of host-parasite Population Interactions: I. Regulatory processes", The Journal of Animal Ecology, 47 (1), pp. 219-247, 1978.

[3] Driessche, Van. Den. and Watmough, P. J.: "Reproduction Numbers and Sub-threshold Endemic Equilibria for Compartmental Models of Disease Transmission", Mathematical Bioscience, 180 (1-2), pp. 29-48, 2002.

[4] Gorman, D. M. et. al.: "Agent-Based Modeling of Drinking Behavior: a Preliminary Model and Potential Applications to Theory and Practice", American Public Health Association, 96 (11), pp. 2055-2060, 2006.

[5] Huo, H. F. and Song, N. N.: "Global Stability for a Binge Drinking Model with Two Stages", Discrete Dynamics Nature and Society, 2012, pp. $1-15,2012$.

[6] Kot, M.: "Elements of Mathematical Biology", Cambridge University Perss: Cambridge, pp. 453, 2001.

[7] Li, M. Y. and Muldowney, J. S.: "A Geometric Approach to Global Stability Problem", SIAM Journal on Mathematical Analysis, 27,(4), pp. 1070-1083, 1996.

[8] Lee, S. et. al: "Optimal Control Intervention Strategies in Low-and High Risk Problem Drinking Populations", Socio-Economic Planning Sciences, 44(4), pp. 258-265, 2010.

[9] Manthey, J. L. et. al.: "Campus Drinking: An Epidemiological Model", Journal of Biological Dynamics, 2 (3), pp. 346-356, 2008.

[10] Mubayi, A. et. al.: "The Impact of Relative Residence Times on the Distribution of Heavy Drinkers in Highly Distinct Environments", SocioEconomic Planning Sciences, 44 (1), pp. 45-56, 2010.

[11] Mulone, G. and Straughan, B.: "Modeling Binge Drinking", International Journal of Biomathematics, 5(1), pp. 1-14, 2012.

[12] Orford, J. et. al.: "University Student Drinking: The Role of Motivational and Social Factors", Drugs: Education, Prevention and Policy, 11(5), PP. 407-421, 2004.

[13] Sharma, S., and Samanta, G. P.: "Drinking as an epidemic: A mathematical Model with Dynamics Behavior", Journal of Applied Mathematics \& Informatics, 31(1-2), pp. 1-25, 2013.

[14] Sharma, S. and Samanta, G. P.: "Analysis of a Drinking Epidemic Model", International Journal of Dynamics and Control, 3 (3), pp. 288-305, 2015.

[15] Wiggins, S.: "Introduction to Applied Nonlinear Dynamical Systems and Chaos", Texts in Applied Mathematics (2nd edition), Springer-Verlag, New York, 2003.

[16] Xiang, H., Song, N. and Huo, H. F.: "Modelling Effects of Public Health Educational Campaigns on Drinking Dynamics", Journal of Biological Dynamics, 10 (1), pp. 164-178, 2016.

[17] Xiang, H., Zhu, C. C. and Huo, H. F.: "Modelling the Effect of Immigration on Drinking Behaviour", Journal of Biological Dynamics, 11(1), pp. 275-298, 2017. 